\title{
cBid, Bax and Bcl-xL exhibit opposite membrane remodeling activities
}

\author{
S Bleicken ${ }^{1,2,3}$, G Hofhaus ${ }^{4}$, B Ugarte-Uribe ${ }^{1,2,3}$, R Schröder ${ }^{4}$ and AJ García-Sáez ${ }^{\star, 1,2,3}$
}

The proteins of the $\mathrm{Bcl}-2$ family have a crucial role in mitochondrial outer membrane permeabilization during apoptosis and in the regulation of mitochondrial dynamics. Current models consider that Bax forms toroidal pores at mitochondria that are responsible for the release of cytochrome c, whereas Bcl-xL inhibits pore formation. However, how Bcl-2 proteins regulate mitochondrial fission and fusion remains poorly understood. By using a systematic analysis at the single vesicle level, we found that $c B i d$, Bax and $\mathrm{Bcl}-\mathrm{xL}$ are able to remodel membranes in different ways. CBid and Bax induced a reduction in vesicle size likely related to membrane tethering, budding and fission, besides membrane permeabilization. Moreover, they are preferentially located at highly curved membranes. In contrast, Bcl-xL not only counterbalanced pore formation but also membrane budding and fission. Our findings support a mechanism of action by which $\mathrm{cBid}$ and Bax induce or stabilize highly curved membranes including nonlamellar structures. This molecular activity reduces the energy for membrane remodeling, which is a necessary step in toroidal pore formation, as well as membrane fission and fusion, and provides a common mechanism that links the two main functions of Bcl-2 proteins.

Cell Death and Disease (2016) 7, e2121; doi:10.1038/cddis.2016.34; published online 25 February 2016

Apoptosis is a form of programmed cell death that removes unhealthy or unnecessary cells from multi-cellular organisms. ${ }^{1-3}$ The mitochondrial pathway of apoptosis results in the permeabilization of the mitochondrial outer membrane (MOM) and in the fragmentation of the tubular mitochondrial network. The members of the Bcl-2 family are crucial in both processes. ${ }^{4-10}$ They are classified into the following three sub-groups: the proapoptotic Bax-type proteins that mediate MOM permeabilization; the anti-apoptotic family members that promote cell survival by inhibiting their pro-apoptotic counterparts; and the BH3-only proteins that promote apoptosis either by inhibiting the antiapoptotic $\mathrm{Bcl}-2$ proteins or by activating Bax and Bak. 7,10

Here, we focused on cBid, Bax and Bcl-xL as representative members of all three subgroups. They shuttle between soluble and MOM-inserted conformations. ${ }^{1-14}$ Apoptosis induction leads to their extended association with the mitochondrial membrane, where Bax and $\mathrm{Bcl}-\mathrm{xL}$ adopt membrane-embedded conformations, ${ }^{14-23}$ and associate into homo- or heterodimers. ${ }^{15-17,21,24-27}$ Moreover, the Bcl-2 proteins are important regulators of mitochondrial morphology. ${ }^{6,28-31}$ Although active Bax promotes mitochondrial fission during apoptosis, ${ }^{6,28-30,32}$ inactive Bax is required for the normal rate of mitochondrial fusion. ${ }^{29}$ Interestingly, Bax co-localizes with proteins involved in mitochondrial fission and fusion (Drp1 and Mfn2). ${ }^{28}$ In addition, $\mathrm{BCl}-\mathrm{xL}$ impacts on mitochondrial fission and fusion, ${ }^{31}$ and stimulates the GTPase activity of Drp1. ${ }^{33,34}$ However, the molecular mechanism underlying these functions remains obscure.
Membrane fission and fusion are lipid-mediated events that proceed through highly curved and energetically unfavorable, non-lamellar membrane structures. ${ }^{35-38}$ The formation of toroidal pores, like those formed by Bax and Bak, ${ }^{39-44}$ involves similar structures. ${ }^{45,46}$ As the $\mathrm{Bcl}-2$ proteins affect both toroidal pore formation and mitochondrial dynamics, it is a reasonable hypothesis that both processes are promoted by a common underlying activity. However, the relationship between these membrane structures and the $\mathrm{Bcl}-2$ proteins remains enigmatic. To tackle this issue, reconstituted model systems have proven useful to learn about the molecular mechanisms involved.

Here, we used minimal systems to investigate how Bcl-2 proteins affect the organization of lipid membranes mimicking the MOM and specifically membrane remodeling. Our results show that cBid alone, cBid/Bax or heat-activated Bax are able to induce or stabilize curved structures, whereas permeabilization requires the presence of Bax. These alterations are specific, as they are abolished by $\mathrm{Bcl}-\mathrm{xL}$. Our findings suggest that the functional roles of $\mathrm{Bcl}-2$ proteins in MOM permeabilization and mitochondrial shape are linked by their ability to stabilize unfavorable membrane structures.

\section{Results}

We previously used model membranes to understand the interaction of $\mathrm{Bcl}-2$ proteins with membranes. ${ }^{14-16,24,43}$ These systems recapitulate the main traits of the biological process,

\footnotetext{
${ }^{1}$ Membrane Biophysics, Max Planck Institute for Intelligent Systems, Heisenbergstrasse 3, Stuttgart 70569, Germany; ${ }^{2}$ German Cancer Research Center, Im Neuenheimer Feld 267, Heidelberg 69120, Germany; ${ }^{3}$ Membrane Biophysics, Interfaculty Institute of Biochemistry, Eberhard Karls University Tübingen, Hoppe-Seyler-Strasse 4, Tübingen 72076, Germany and ${ }^{4}$ CellNetworks, Bioquant, Heidelberg University, Im Neuenheimer Feld 267, Heidelberg, 69120, Germany

${ }^{*}$ Corresponding author: AJ García-Sáez, Membrane Biophysics, University of Tuebingen, IFIB, Hoppe-Seyler-Strasse 4, Tübingen 72076, Germany. Tel: +49 70712973318; Fax: +49 7071295070; E-mail: ana.garcia@uni-tuebingen.de

Abbreviations: APC, Allophycocyanin; FCS, fluorescence correlation spectroscopy; Cyt c, Cytochrome c; DLS, dynamic light scattering; EM, electron microscopy; GUVs, giant unilamellar vesicles; LUVs, large unilamellar vesicles; MOM, mitochondrial outer membrane

Received 18.9.15; revised 20.1.16; accepted 25.1.16; Edited by G Dewson
} 
while decreasing its complexity so that aspects like pore formation or membrane fusion and fission can be studied in more detail than it is possible in cells. Here, we systematically investigated the effect of different $\mathrm{Bcl}-2$ proteins on membrane structure. As the biophysical methods used have different requirements in terms of membrane amounts, we have kept the protein to lipid ratio constant to make the experiments comparable (Bax to lipid ratio 1: 500-1000; cBid to Bax ratio 1 :2; $\mathrm{Bax}$ to $\mathrm{Bcl}-\mathrm{xL}$ ratio $2: 5$ ). Unless otherwise stated, we used a lipid mixture mimicking the MOM that is well established in the field. ${ }^{16,21,47}$

Giant unilamellar vesicle (GUV) permeabilization was followed by the entry of soluble, fluorescently labeled proteins into the liposomes (introduced in Bleicken et al. ${ }^{43}$ ). After incubation in absence or presence of $\mathrm{Bcl}-2$ proteins, the vesicle suspensions were imaged and analyzed with a homebuilt software ${ }^{48}$ to quantify GUV size, shape and permeabilization. To detect membrane remodeling, we analyzed the median radius of the GUVs and normalized it by the median radius of the control GUVs. The radii of the GUVs considered in the experiments ranged between 2 and $50 \mu \mathrm{m}$. Smaller GUVs could not be detected due to the resolution of the method, whereas bigger GUVs were rare. The impact of different $\mathrm{Bcl}-2$ proteins is expressed relative to the control.

To validate the method, the influence of Drp1 on the GUV radius was tested. In vivo, Drp1 is involved in mitochondrial fission $^{49,50}$ and in vitro it induces tube formation and vesicle tethering. ${ }^{40,51}$ Both changes extract membrane material and are expected to lead to a reduction in GUV size, as detected experimentally (Figures 1a and c-e for 5 nM Drp1; Figure 2d for $10 \mathrm{nM}$ Drp1). Drp1 induced the formation of membrane tubes and buds on the GUV surface, which contrasted with the more homogeneous surface of control GUVs (Figures 1c and e). These effects were specific for Drp1, as GTP addition slightly enhanced GUV size reduction induced by Drp1, whereas addition of the Drp1 inhibitor mdivi-1 ${ }^{52,53}$ decreased the effects (Figures 1a, $d$ and e). We did not detect vesicle bursting neither did Drp1 induce GUV permeabilization (Figure 1b), demonstrating that permeabilization and size reduction are not necessarily coupled.

cBid and Bax cause membrane permeabilization and a reduction in GUV size, while both processes can be inhibited by $\mathbf{B c l}-\mathbf{x L}$. We then investigated the effect of individual $\mathrm{Bcl}-2$ proteins on GUV size. Bax or $\mathrm{Bcl}-\mathrm{xL}$ alone do not spontaneously bind to the membranes in the absence of $\mathrm{cBid}^{21,24,26}$ and thus they did not affect GUV size nor did they induce membrane permeabilization (Figures $2 b$ and $d$ ). However, cBid mixed with Bax caused a significant reduction in GUV size and membrane permeabilization (Figures $2 b, d$ and $3 b)$.

Surprisingly, also cBid alone caused a concentrationdependent reduction of GUV size (Figures $2 d$ and $3 b$ ), whereas no significant membrane permeabilization was detected (Figures 2a and b). The effect of cBid was specific, as Bcl-xL completely neutralized this effect despite enhancing cBid membrane binding ${ }^{24,26}$ (Figures $2 d$ and $3 b$ ). In presence of all three proteins, mild membrane permeabilization and size reduction was detected (Figures $2 b$, $d$ and $3 b$ ), showing that
$\mathrm{Bcl}-\mathrm{xL}$ strongly counterbalanced the effects of $\mathrm{cBid}$ and Bax in line with its inhibitory role.

To separate the membrane effects of Bax and $\mathrm{cBid}$, we tested an alternative method to activate Bax using mild heat $\left(43^{\circ} \mathrm{C}\right) .{ }^{48,54}$ This treatment led to substantial membrane permeabilization in presence of Bax, but not in the control (Figure 2c), similar to our observations with cBid-induced Bax pores $^{43}$ (Figure 2b). Moreover, heat-activated Bax also reduced the median GUV size (Figure $2 \mathrm{e}$ ).

In addition, we used an alternative assay to detect vesicle size reduction based on large unilamellar vesicles (LUVs) and fluorescence correlation spectroscopy (FCS). The diffusion of LUVs through the focal volume of the confocal microscope is detected and analyzed by an auto-correlation analysis. The vesicle diffusion time, which directly relates to the vesicle size, is reflected in the decay of the auto-correlation curve, whereas the amplitude is inversely proportional to the concentration of vesicles. In Figure $2 \mathrm{f}$, the auto-correlation curves of a representative experiment are shown, while Figures $2 \mathrm{~g}$ and $\mathrm{h}$ depicts the changes in vesicle diffusion time and particle numbers averaged from three independent experiments.

LUVs incubated with cBid or cBid/Bax showed a significantly shorter diffusion time and an increased concentration compared with the control sample (Figures $2 \mathrm{~g}$ and $\mathrm{h}$ ), which indicates a reduction in vesicle size as in the GUV experiments. Moreover, cBid and cBid/Bax increased the number of vesicles, suggesting vesicle fission. $\mathrm{Bcl}-\mathrm{xL}$ inhibited both the changes in vesicle size and number (Figures $2 g$ and $h$ ).

cBid and Bax promote vesicle budding and fission. These data showed that cBid, Bax and Bcl-xL changed membrane structure beyond pore formation. The FCS experiments suggested vesicle fission involved. If this was the case, buds should be formed on the GUV surface, which we visualized with confocal microscopy. In absence of Bcl-2 proteins, the z-projections of GUVs showed a homogenous vesicle surface, but incubation with $\mathrm{cBid}$ and Bax induced bright dots on the surface (Figures 1c and e), which have been previously associated with membrane buds. ${ }^{55-57}$ In some cases we were able to image bud formation and fission (mainly happening towards the outside of the GUV's; Figure $3 a$ ). However, due to its rare and stochastic nature, bud formation and release was difficult to follow by imaging.

Compared with the control, images of GUVs incubated with cBid or cBid/Bax showed an increase in small floating particles (Figure 2a), likely related to released buds. In order to quantify the membrane material dissociated from the GUVs, the detection volume of the confocal microscope was placed above the GUVs and fluorescence intensity traces were recorded. Diffusing vesicles appear as peaks in the intensity traces (Figure $3 c$ ). To separate peaks corresponding to bud particles from noise and background fluorescence, we set a threshold 10 times bigger than the baseline of background fluorescence (Supplementary Information). In parallel, we followed the changes in GUV size, as budding should correlate with a decrease in GUV size (Figure $3 b$ ).

Compared with the control, cBid or cBid/Bax provoked a clear and concentration-dependent increase in the number of peaks (Figure $3 d$ ), which correlate with lipid particles being extracted from the peaks showed a broad range of intensities 


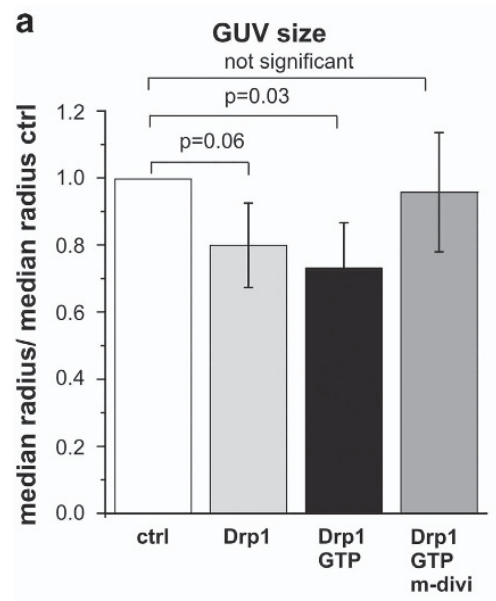

d 5 nM Drp1

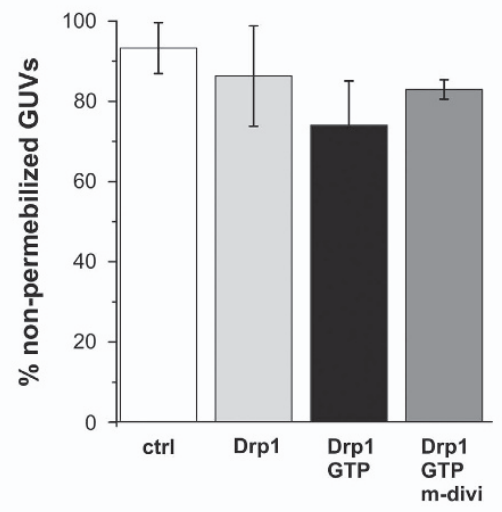

C ctrl
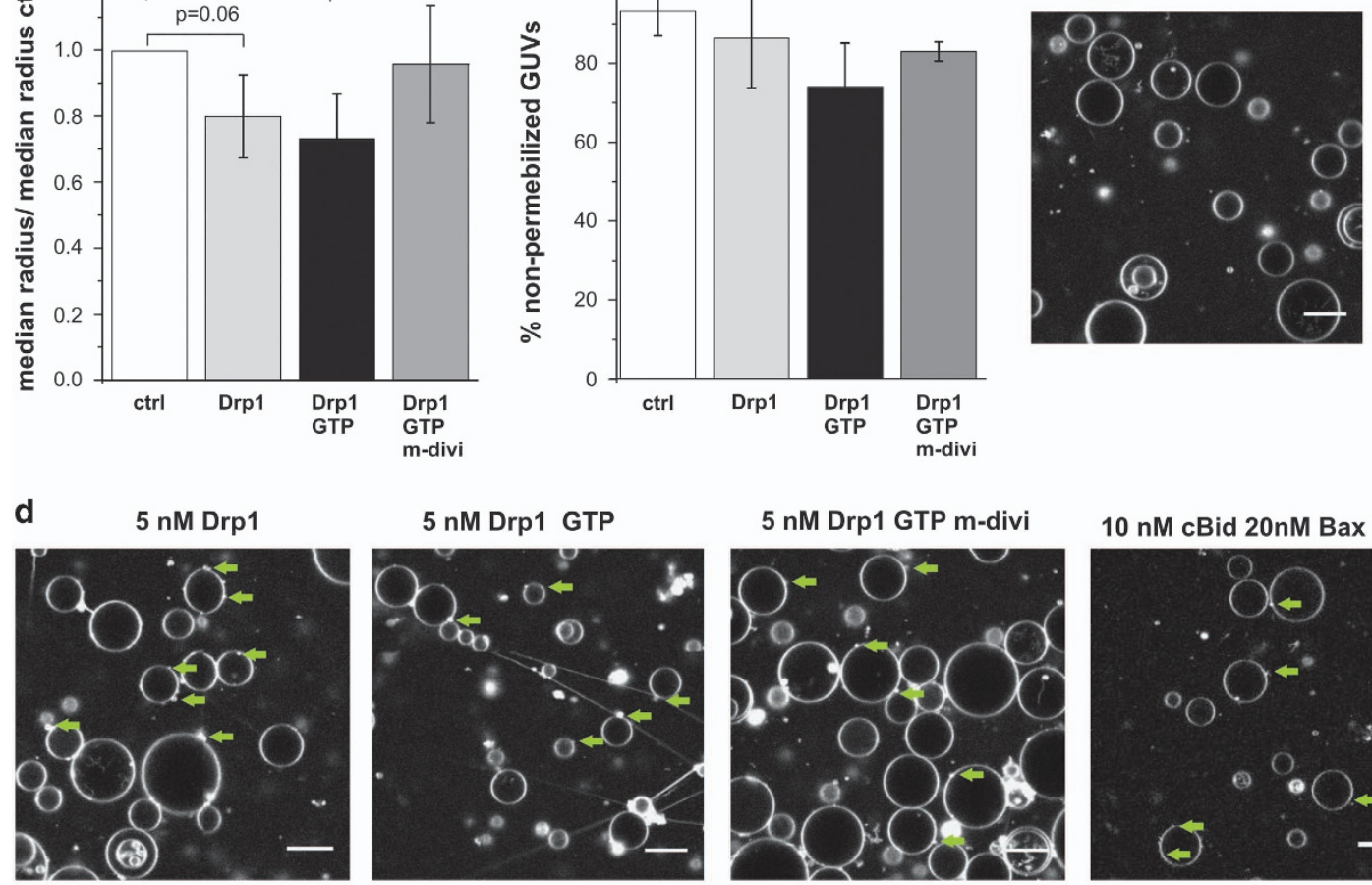

5 nM Drp1 GTP

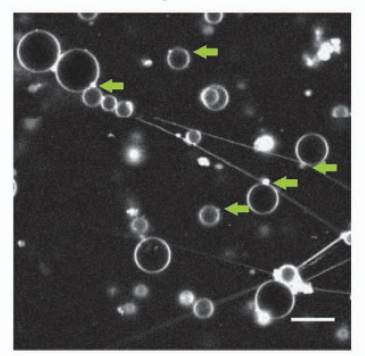

5 nM Drp1 GTP m-divi

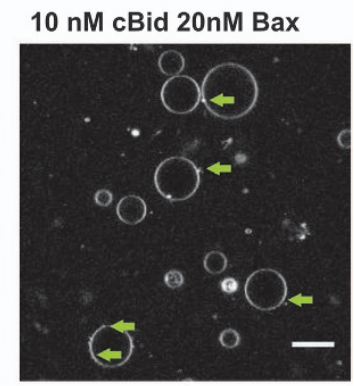

e ctrl

5 nM Drp1
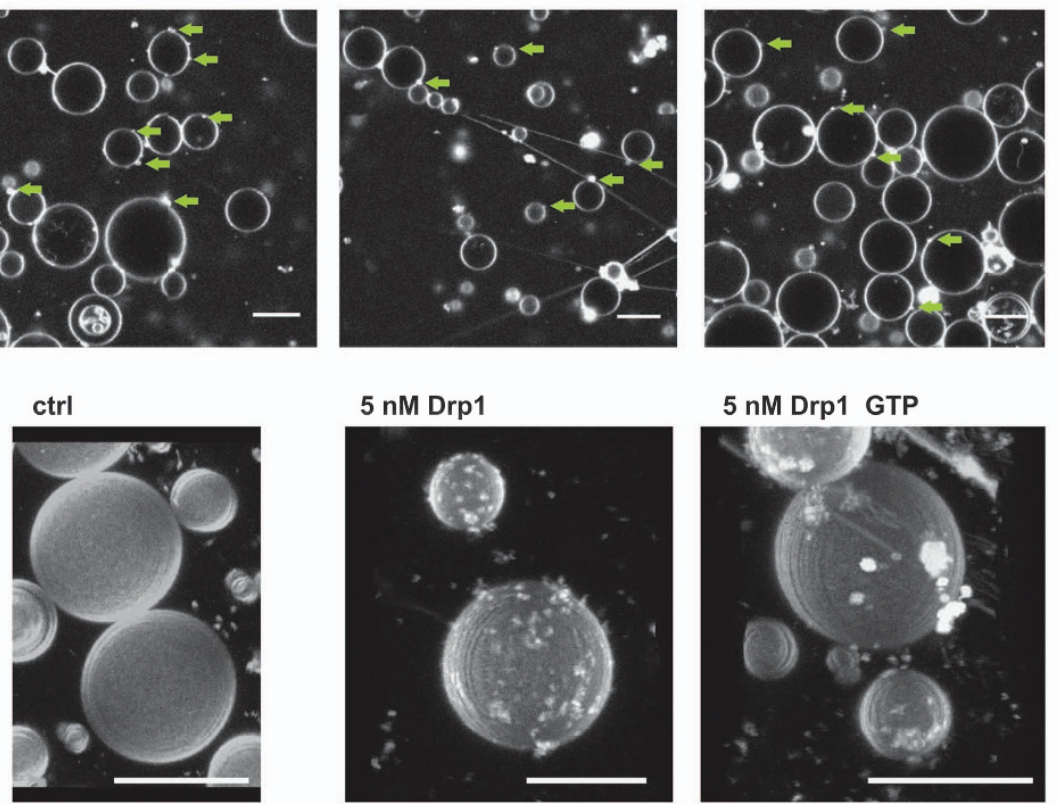

5 nM Drp1 GTP

10 nM cBid 20nM Bax
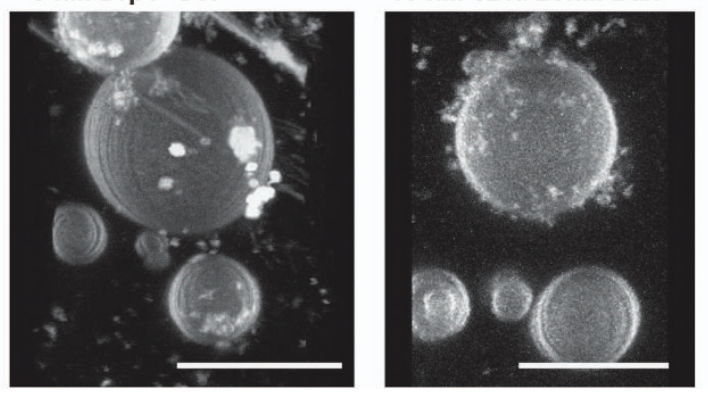

Figure 1 Changes in GUV size upon incubation with Drp1. (a) Comparison of the median GUV radius from GUV incubated 90 min with only Cyt $\mathrm{C}_{488}$ (control) or additionally with $5 \mathrm{nM}$ Drp1, $1 \mathrm{mM}$ GTP or $50 \mu \mathrm{m}$ mdivi-1 normalized to the median radius of the control sample. Notably, mdivi-1 partly precipitated, thus the effective mdivi-1 concentration is lower as $50 \mu \mathrm{M}$. Error bars in (a) and (b) correspond to the S.D. $(N=3)$. (b) Percentage of non-permeabilized GUVs for Cyt $\mathrm{C}_{488}$ from (a). (c-e) Confocal images (c and d) and 3D reconstitution of z-stacks from GUV incubated with the size marker proteins alone or with the components indicated in the figure. In all experiments the GUVs were composed of a lipid mixture mimicking the MOM and labeled with $<0.05 \%$ Dil. Green arrows in (d) indicate structures indicative for vesicle buds. Scale bar: $20 \mu \mathrm{m}$

(Figure 3e) and residence times (Figure 3c) indicating particles of different sizes. Notably, the presence of $\mathrm{Bcl}-\mathrm{xL}$ had a clear inhibitory effect on the process (Figure 3d).

Bigger vesicles need longer times to cross the focal volume and are brighter due to the larger amount of fluorophores they contain. We calculated the average times differently sized vesicles need to cross the focal volume and the expected brightness of those particles (Supplementary Information). From the imaging (Figures 1d and e), we know that the buds have mainly a radius below $1 \mu \mathrm{m}$, which would correspond to residence times $<0.3 \mathrm{~s}$ and brightness $<1000 \mathrm{kHz}$ (Supplementary Information), which fits well with the experimental data (Figures $3 c$ and e). A few brighter particles with longer residence times are detected, which are mainly present upon incubation with cBid/Bax (Figure 3e). These particles could be tubes, GUVs or tethered vesicles with slow diffusion.

cBid and Bax bind to highly curved membranes. Bud and GUV are connected by a highly curved neck region (Figure 4a) and toroidal pores are also characterized by highly curved membranes (Figure 4b). To compare the localization of cBid and Bax in curved versus flat parts of the membrane, GUVs enriched in cardiolipin $(30 \% \mathrm{CL}$ and $70 \%$ PC) were incubated with fluorescently labeled cBid and Bax. The label did not interfere with protein action ${ }^{24}$ and the lipid mixture was to be suitable to study pore formation and membrane binding. ${ }^{24,43}$ The resolution of the confocal microscope was too low to discriminate whether the protein 
binds to the bud or the contact area of small buds. Therefore, the analysis was limited to a subpopulation of relatively big buds. cBid and Bax were found predominantly in the neck between vesicle and bud (Figures $5 \mathrm{a}-\mathrm{c}$ ). The fluorescence intensity was higher than expected for two membranes
(Figures $5 \mathrm{c}$ and $\mathrm{f}$ ), demonstrating that $\mathrm{cBid}$ and Bax were enriched in the neck region. To ensure that the bright areas contained membrane and were not protein aggregates, GUVs labeled with a green fluorescent dye and Bax Atto655 $_{5}$ (activated by unlabeled cBid). This showed that the mem-

a example images

ctrl

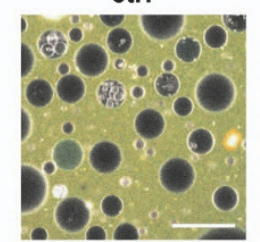

b

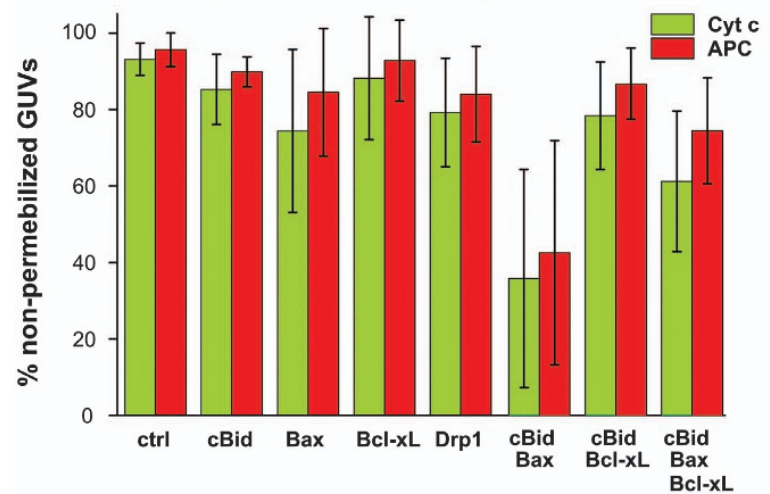

cBid/Bcl-xL

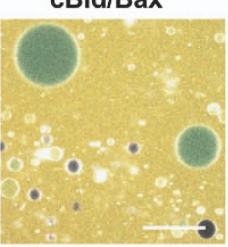

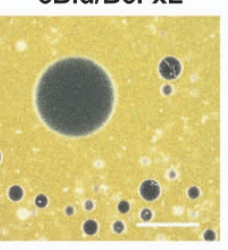

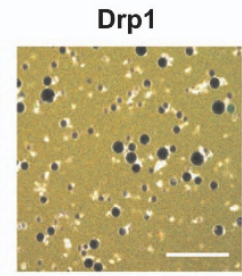

c

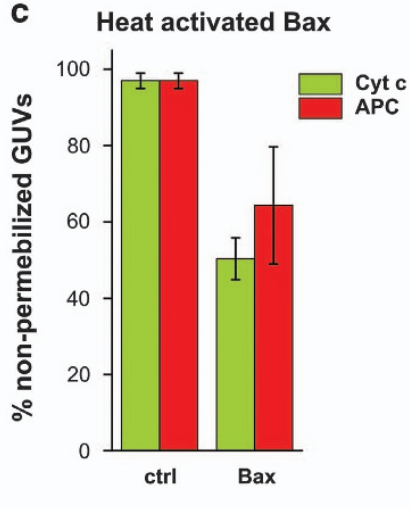

d

Bcl-2 protein induced changes in GUV size
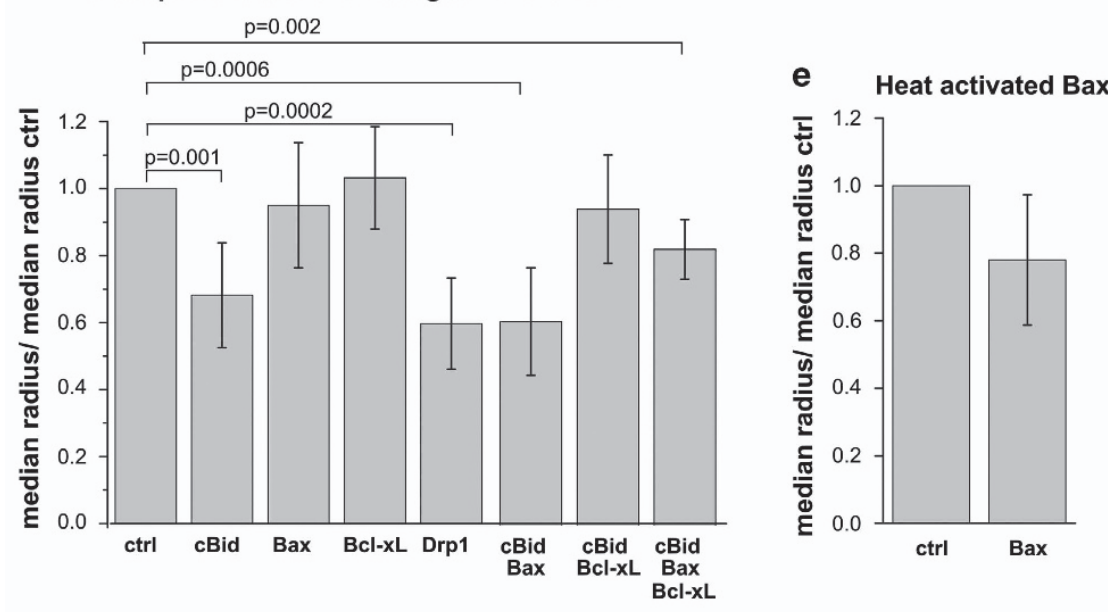

f

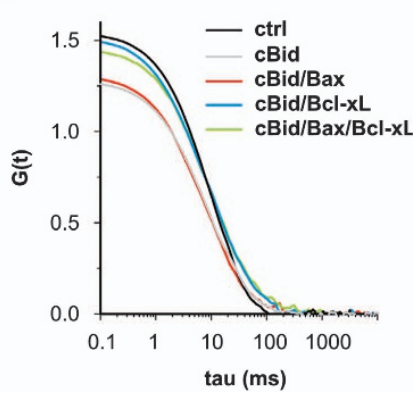

g

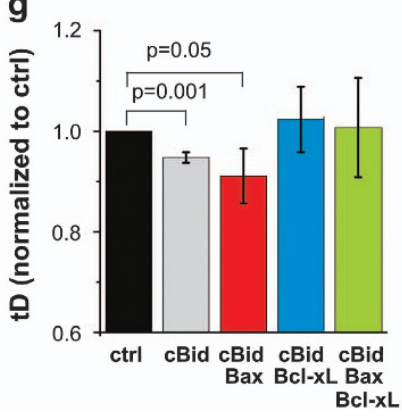

h

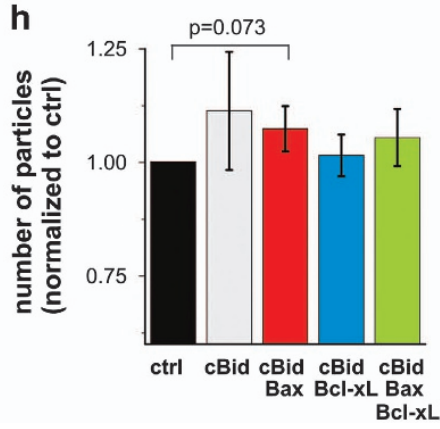


brane is present at the neck and that Bax, but not the membrane dye was highly enriched in that area (Figure $5 \mathrm{~g}$ ).

cBid/Bax induce membrane tethering and pore formation in LUVs visualized by cryo-EM. The spatial resolution of confocal microscopy does not allow following cBid/Baxinduced morphological changes at the membrane with molecular detail. To overcome this limitation, we used cryo electron microscopy (EM). As the ice layer of a cryo grid is typically a few-hundred nanometers thick, the micrometersized GUVs would be destroyed or strongly distorted by the blotting and we could not get images of them. Instead we used LUVs of $100-400 \mathrm{~nm}$ diameter (Figure 6e) with the disadvantage that the LUV's have a different curvature as the GUVs, the proteins might not act the same way.

Membrane pores induced by Bax with diameters between 10 and $>100 \mathrm{~nm}$ were previously imaged using cryo EM. ${ }^{15,58-60}$ We detected similar pores with negative lipid curvature at the pore edges, in line with a recent publication ${ }^{60}$
(Figure 6a). Like the previous studies, ${ }^{15,58-60}$ we could not detect electron densities related to the proteins. This is compatible with our 'clamp' model of membrane-embedded Bax, in which Bax lies on the membrane surface to stabilize the toroidal pore ${ }^{16}$ (Figure $4 \mathrm{~b}$ ) and could make it hard to discriminate electron density related to the protein from the membrane.

Aside from the pores, we observed a higher percentage of tethered vesicles in presence of $\mathrm{cBid}$ and Bax compared with controls (Figure 6b). Vesicle tethering is a necessary structural intermediate of membrane fission and fusion (Figure 4a), and was detected in EM studies of vesicle fusion. ${ }^{61,62}$ As Drp1 has also been shown to induce membrane tethering, ${ }^{40,51}$ it is reasonable to hypothesize that the membrane tethering induced by $\mathrm{cBid} / \mathrm{Bax}$ might be related to membrane remodeling.

To study the temporal evolution of pore formation and tethering, we compared vesicles incubated $10 \mathrm{~s}$ to $60 \mathrm{~min}$ with $\mathrm{cBid} / \mathrm{Bax}$ with control vesicles and counted the fraction of vesicles showing pores or tethering. To have a suitable

\section{a Kinetic experiments}
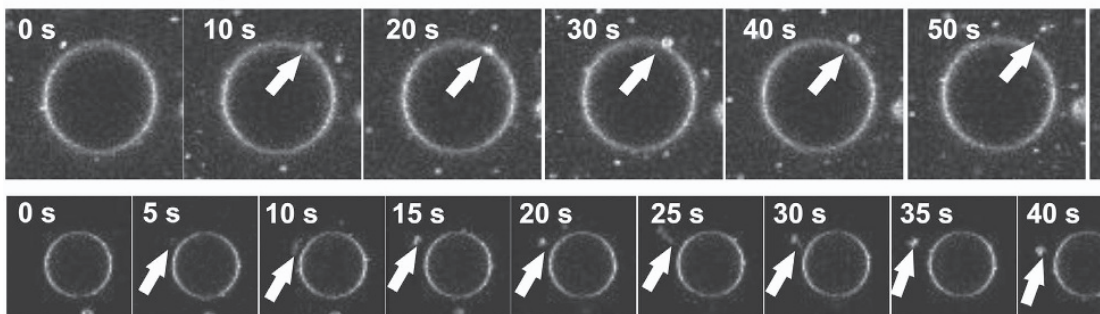

d

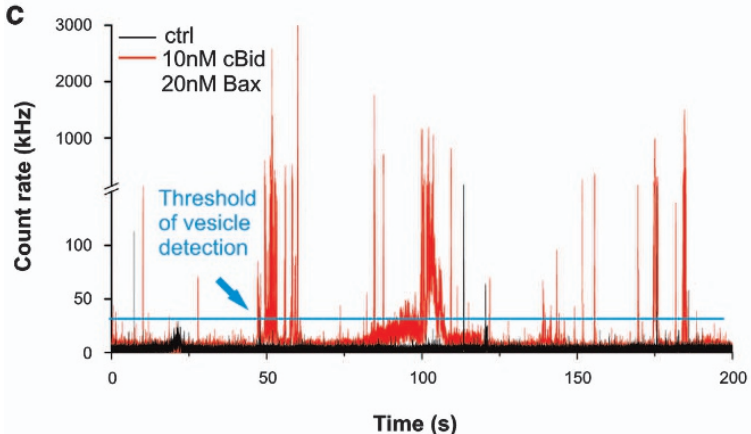

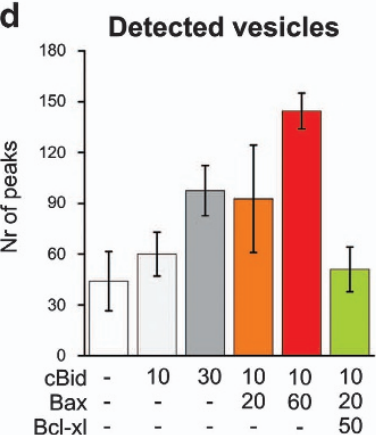

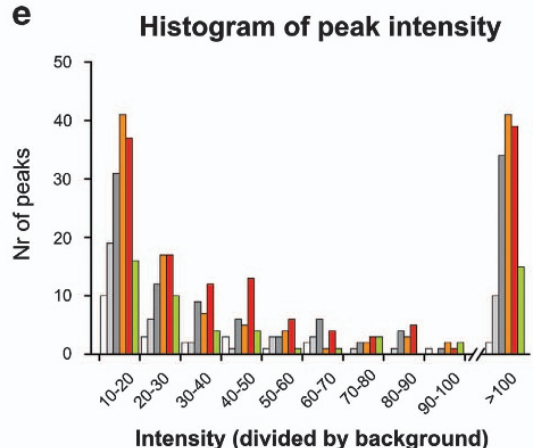

Figure 3 GUV budding upon incubation with Bcl-2 proteins. (a) Snapshots of vesicle budding from kinetic experiments. GUV mimicking the MOM composition labeled with Dil incubated with $10 \mathrm{nM} \mathrm{cBid}$ and $20 \mathrm{nM}$ Bax. Scale bar: $20 \mu \mathrm{m}$. (b) Changes in GUV size upon 90 min incubation with the indicated proteins at $22^{\circ} \mathrm{C}$. (c) Exemplary fluorescence intensity trace of a control sample and a sample incubated with $10 \mathrm{nM}$ cBid and $20 \mathrm{nM} \mathrm{Bax}$. The background count rate is $<3 \mathrm{kHz}$. Fluorescent bursts $>10$ times brighter as the background count rate are considered as diffusing vesicles. (d) Number of peaks detected during $600 \mathrm{~s}$ (with count rates $>29 \mathrm{kHz}$ ) after 90 min incubation with the indicated proteins at $22{ }^{\circ} \mathrm{C}$. Error bar present the S.D. $(N=3)$. (e) Histogram of the different peak intensities describes in (d). The color code is the same as in (d)

Figure 2 Changes in GUV size and membrane permeabilization induced by Bcl-2 proteins. (a) Representative images of GUVs (gray) in a solution of Cyt $\mathrm{C}_{488}(12 \mathrm{kDa}$, green) and allophycocianin (APC) (104 kDa, red) incubated in the absence (ctrl) or in the presence of $10 \mathrm{nM} \mathrm{cBid,} 20 \mathrm{nM} \mathrm{Bax}, 50 \mathrm{nM} \mathrm{Bcl-xL}, 10 \mathrm{nM}$ Drp-1 or combinations of these proteins. Scale bar: $75 \mu \mathrm{m}$. (b and $\mathbf{c}$ ) Fraction of non-permeabilized GUVs in the control sample or in presence of the indicated proteins or protein mixture (b) or after heat treatment (c). Green and red bars correspond to Cyt $\mathbf{c}_{488}$ and APC, respectively. (d and $\left.\mathbf{e}\right)$ Comparison of the median GUV radius from the experiments introduced in (b and $\mathbf{c}$ ) normalized to the median radius of the control sample $(4.97 \pm 1.07 \mu \mathrm{m})$. (d) Results for individual proteins or protein mixtures. (e) Results from heat-treated samples. In each of four independent experiments, a minimum of 200 vesicles were analyzed per condition. Error bars represent the S.D. (f- $h$ ) Fluorescence correlation spectroscopy experiments on vesicle diffusion after $2 \mathrm{~h}$ incubation in buffer or with the indicated Bcl-2 proteins. Error bars present the S.D. (f) Auto-correlation curves from one exemplary experiment. The color code is indicated within the figure. ( $\mathbf{g}$ and $\mathbf{h}$ ) Comparison of the vesicle diffusion times $(\mathrm{tD})(\mathbf{g})$ and particle numbers $(\mathbf{h})$ from three independent experiments normalized to the tD or particle number of the control vesicles. Error bars correspond to the S.D. To achieve the tD a 3D fit was used 
a

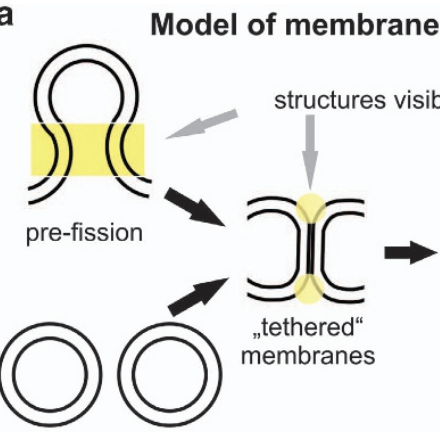
pre-fusion b

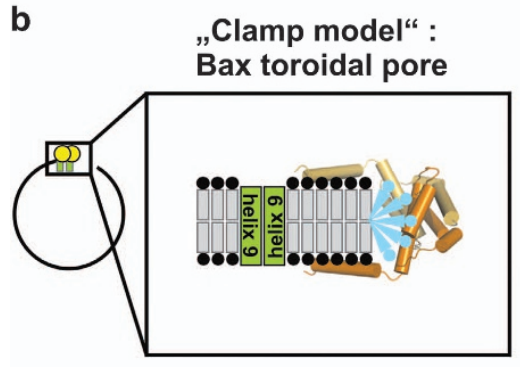

\section{fission and fusion}

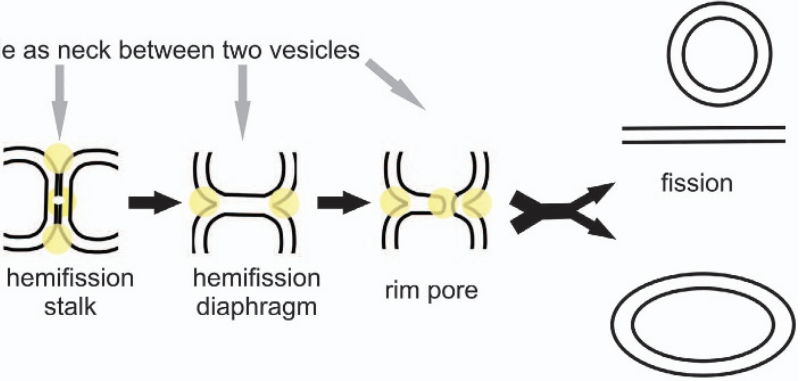

fusion

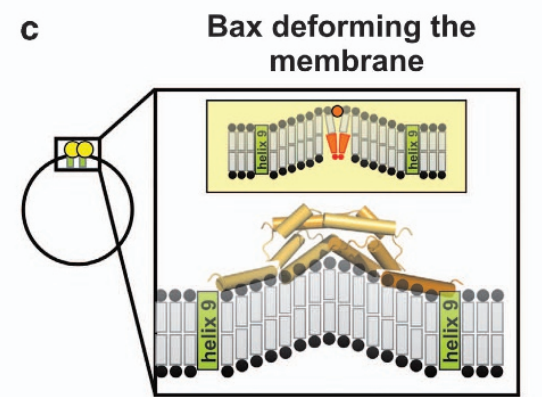

Figure 4 Model of membrane fission and fusion, and possible scenario on how Bax reorganizes the membrane. (a) Model of membrane fission and fusion was adapted from Frolov and Zimmerberg. ${ }^{35}$ Fission and fusion are lipid-mediated events that are mainly described theoretically ${ }^{35-38}$ and are proposed to happen via the same intermediates. The membrane that fuse or divide needs to come into close contact, allowing the formation of a so-called 'hemi-fission/fusion stalk'. Stalk formation is proposed to start via exchange of lipid tails at the contact area of the two outer membrane leaflets. This creates a connection that may grow into a 'hemi-fission/fusion diaphragm'. However, this process is energetically unfavored and needs the exclusion of water molecules from the contact area. Therefore, the membranes need to come in close contact, which are visible as membrane tethering. To resolve the 'hemi-fission/fusion diaphragm' into the fused or divided membranes, the opening of a transient pore (called the 'toroidal rim pore') has been proposed. As a result, membrane fusion and fission proceed via several unstable structures containing highly curved membranes (highlighted in yellow), which can happen spontaneously, but in cells is usually stabilized by dedicated proteins. (b) 'Clamp model' of membrane embedded Bax stabilizing a toroidal pore. ${ }^{16}$ Toroidal pore formation is eased when the pore rim is formed by lipid having an inverted cone shape (indicated in blue). (c) Suggestions how Bax may induce membrane curvature. Upper example: the shallow insertion of one hydrophobic helix (shown as orange circle) acting like a wedge in the membrane that induces curvature. Here, cone-shape lipids (shown as orange lipid) like cardiolipin can stabilize the membrane curvature. Lower example: similar to BAR domains Bax could force the membrane to bend due to scaffolding. Here Bax is drawn according to the 'in plane' model ${ }^{18}$

number of vesicles the results from all experimental repetitions were merged (Table 1). Due to an insufficient number of LUVs, the 60-min sample was excluded from analysis. The amount of tethered vesicles increased during the first 5-10 min and decreased afterwards. In contrast, the fraction of pore containing vesicles increased over time. Thus, cBid and Bax seemed to promote transient membrane tethering before pore formation (Figures $6 c$ and d; Table 1).

To back up the poor statistics of the cryo EM experiments, we performed bulk experiments using dynamic light scattering (DLS). In time course experiments, control vesicles without protein were constant in size, whereas cBid/Bax induced a transient increase in LUV size during the first minutes of incubation in line with vesicle tethering (Figures $6 e$ and g; Supplementary Figure 1). At later time points, a new population of smaller vesicles was detected in agreement to vesicle fission (Figure 6h). As the increase in vesicle size was transient, vesicle tethering was likely not associated with membrane fusion.

In contrast to the cryo EM results, DLS and FCS showed no indication of vesicle loss upon 1-2 $\mathrm{h}$ incubation with $\mathrm{cBid} / \mathrm{Bax}$ (Figures $2 \mathrm{~h}$ and 6h). As cryo EM samples need to be transferred onto a grid and frozen before image acquisition, it could be that a specific loss of liposomes with membrane bound $\mathrm{cBid} / \mathrm{Bax}$ happened due to changes in the surface properties of the vesicles. In this scenario, the number of pore containing and tethered vesicles would be underestimated, without affecting the conclusion of the experiment.

\section{Discussion}

In cells, Bcl-2 proteins have roles in MOM permeabilization (by forming toroidal pores) and in alterations in mitochondrial dynamics. ${ }^{7,9,63}$ Although the molecular mechanisms involved are poorly understood, a connecting theme between both is the presence of locally highly curved and non-lamellar membrane structures. Here we have used minimal systems to disect the membrane activity of representative $\mathrm{Bcl}-2$ proteins with a focus on membrane remodeling. The usefulness of such strategy is supported by the fact that most of our current understanding about membrane permeabilization, fission and fusion is derived from studies using membrane model systems, ${ }^{21,27,61,64-67}$ as the complexity of cells and the associated technical limitations currently prevent the study of these processes in depth in vivo.

In addition to permeabilization, $\mathrm{cBid}$ and Bax promoted the formation of tethered vesicles, buds on the GUV surface and release of lipid material from the membrane. Remarkably, both membrane permeabilization and budding activity could be 
a

Binding of cBidred and

Baxgreen to GUV

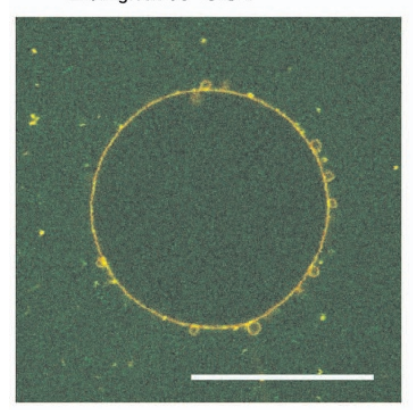

c

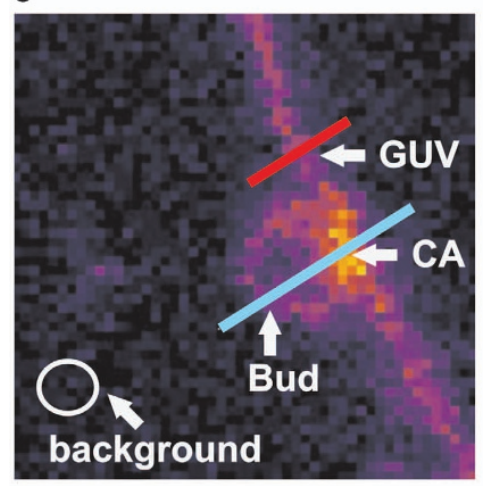

b

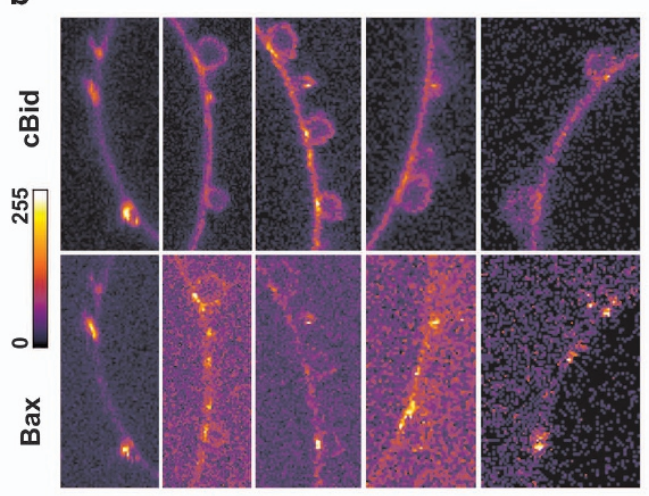

d

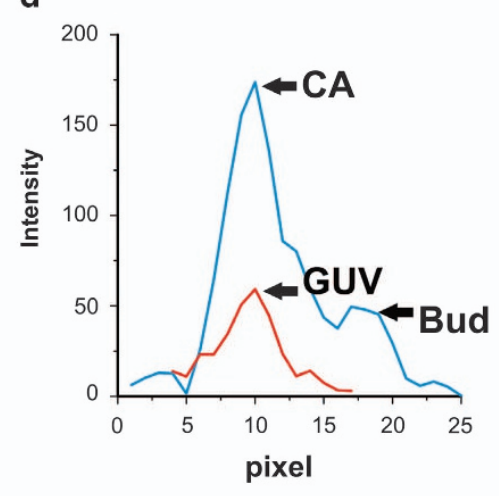

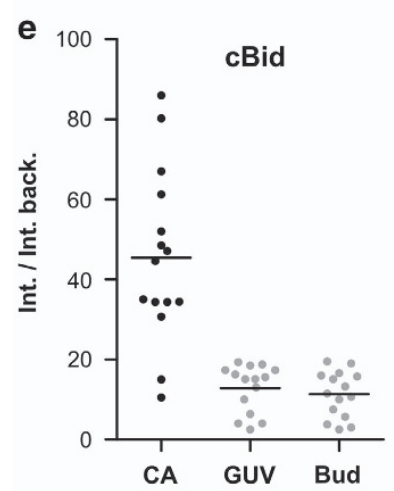
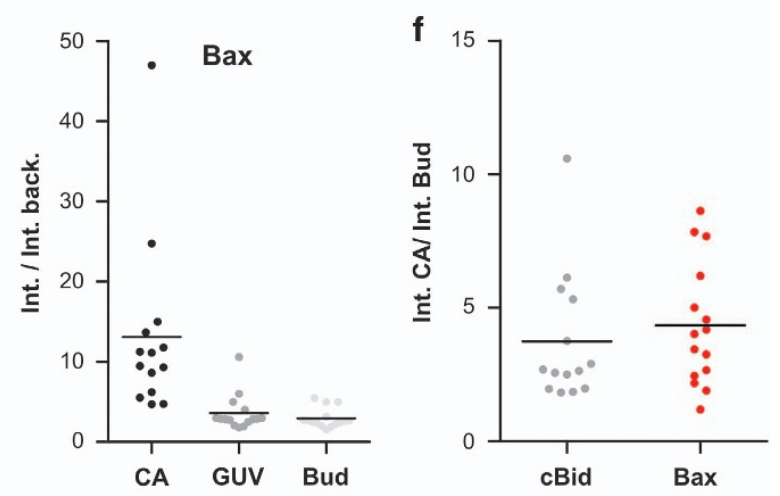

g

DiO

Bax 655
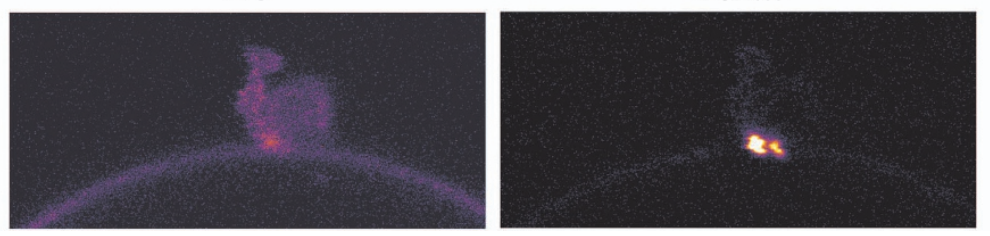

Figure 5 cBid and Bax bind preferentially to highly curved membranes. (a) Image of one GUV composed of $30 \% \mathrm{CL}$ and $70 \% \mathrm{PC}$ after 30 min incubation with $20 \mathrm{nM}$ cBid 655 and $40 \mathrm{nM} \mathrm{Bax}{ }_{488}$. Shown is the color merge. Scale bar: $50 \mu \mathrm{m}$. (b) Zoom-in's from (a) and other GUVs are shown to visualize that cBid and Bax bind preferentially at the highly curved interface between bud and GUV. A scale bar concerning the color code related to the pixel intensity is shown on the left side. (c) Example image on how the pixel intensities in the bud membrane, in the GUV membrane and in the contact area (indicated as CA) as well as the background are measured. The results are drawn in (d)-(f). (d) Display of the exemplary line scans shown in (c). (e) Pixel intensities for cBid and Bax at the contact area, the GUV and the bud (divided by the background intensity) from several GUV/bud pairs. (f) Ratio of the pixel intensity in the GUV/bud contact area compared with the pixel intensities at the GUV membranes. (g) Zoom-in from an exemplary GUV bud pair labeled with DiO and incubated with cBid unlabeled and $40 \mathrm{nM} \mathrm{Bax}_{655}$ 


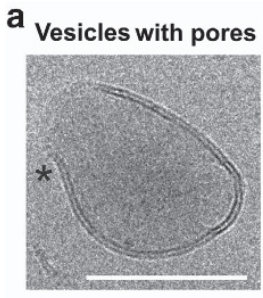

b

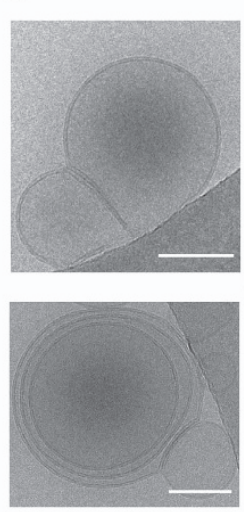

Tethered vesicles

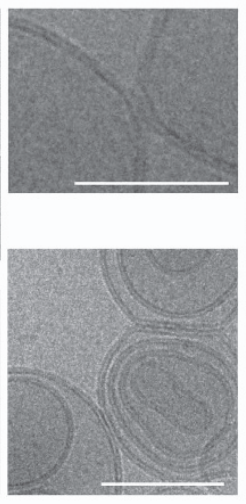

f

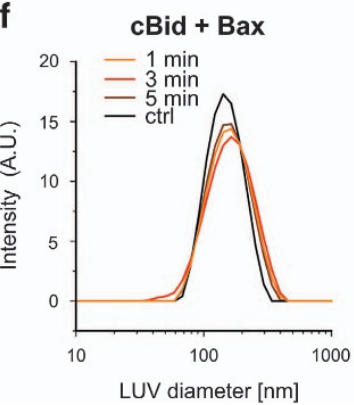

e

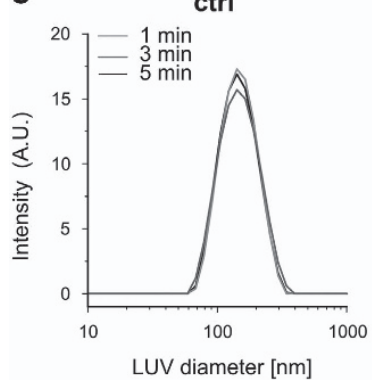

LUV diameter [nm]

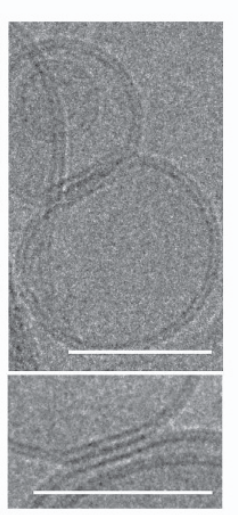

g

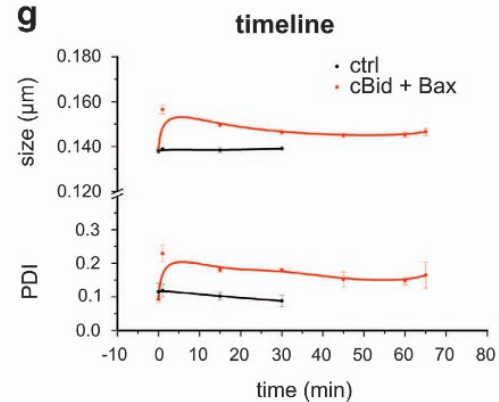

c

d

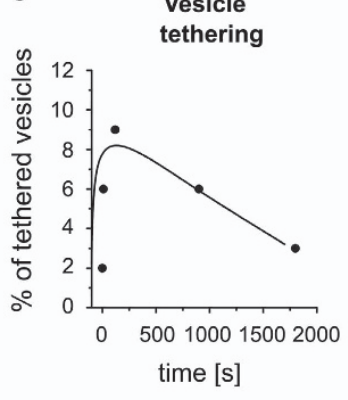

Pore evolution

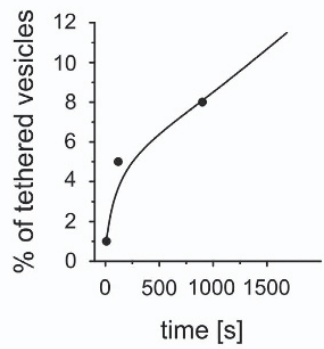

Figure 6 Morphological changes on LUVs upon incubation with cBid/Bax/Bcl-xL. (a and $\mathbf{b}$ ) Cryo EM images of pore containing (asterisk indicates pore edge region with negative curvature as introduced by Gillies et al. ${ }^{60}$ ); (a) or tethered (b) vesicles. Scale bar: $75 \mathrm{~nm}$. (c and d) Percentage of tethered (c) or pore containing (d) vesicles at the different time points gained from Cryo EM experiments. Final concentrations used for cyro EM: $1 \mu \mathrm{M} \mathrm{cBid;} 2 \mu \mathrm{M} \mathrm{Bax} ; 2 \mathrm{mM}$ lipids. For further information on the number of vesicles or experiments see Table 1. (e, $\mathbf{f}$ and $\mathbf{h}$ ) Size distribution of control vesicles (e) or vesicles incubated with cBid and Bax (f and $\mathbf{h}$ ) during different time points of DLS experiments. (g) DLS time course experiment following vesicle size and homogeneity over time. Here one representative experiment of three is shown. Final concentrations used for DLS: 20 nM cBid; 40 nM Bax; $40 \mu$ M lipids

Table 1 Cryo EM image analysis

\begin{tabular}{|c|c|c|c|c|}
\hline & Number of vesicles & $\%$ Unilamilar vesicles & $\%$ Tethered vesicles & $\%$ Pores \\
\hline ctrl & 924 & 70 & 2 & 1 \\
\hline $\mathrm{cBid} / \mathrm{Bax} 10 \mathrm{~s}$ & 988 & 69 & 6 & 1 \\
\hline cBid/Bax 2 min & 722 & 69 & 9 & 5 \\
\hline $\mathrm{cBid} / \mathrm{Bax} 15 \mathrm{~min}$ & 745 & 70 & 6 & 8 \\
\hline $\mathrm{cBid} / \mathrm{Bax} 30 \mathrm{~min}$ & 327 & 86 & 3 & 12 \\
\hline
\end{tabular}

specifically inhibited by $\mathrm{Bcl}-\mathrm{xL}$, indicative of the specific action of cBid and Bax. cBid and Bax were preferentially located at the neck region between GUV and bud, and showed the intrinsic ability to induce or stabilize membrane areas with high curvature and non-lamellar organization. As a consequence, these proteins reduced the energy required for membrane remodeling, which could have a role in the processes of pore formation and membrane fission and fusion.

What do the mechanisms of membrane budding, fission, fusion and pore formation have in common? Budding is the generation of a curved membrane region that can evolve to membrane fission. To start the fission process, the two membranes need to be very close together, which is usually achieved via membrane constriction, followed by the formation of a highly curved, non-lamellar neck region, which evolves to finally separate two membrane-enclosed compartments. Membrane fusion proceeds via the same structural intermediates but in opposite direction, and it is initiated via membrane tethering instead of constriction (Figure 4a). Main drivers of membrane scission are the members of the dynamin superfamily, whereas proteins such as the SNARE proteins are responsible for vesicle fusion. ${ }^{68,69}$ Fusion and scission are favored by high local concentrations of shallowly inserted amphipathic protein helices that create local membrane curvature. $^{66,68}$ These amphipathic helices may be part of the fusion/fission protein structure, like in the case of the $\mathrm{PH}$ domain of dynamin, or of additional protein factors that participate in the process (like synaptotagmin in membrane fusion or epsin in fission). ${ }^{62,66,70,71}$ Drp1 does not contain such a helix and likely needs additional cofactors to mediate 
mitochondrial fission. ${ }^{49}$ Interestingly, the stabilization of local membrane curvature is also crucial for toroidal pores.

Bax and cBid both contain amphipathic helices that have been proposed to superficially interact with membranes ${ }^{16-18,72}$ and could induce local membrane curvature by shallow insertion. The bigger the area of amphipathic helix (helices) is with respect to rest of the protein, the stronger its effect on membrane curvature, which is additionally enhanced by protein oligomerization enhances. ${ }^{66,68,73}$ As cBid and Bax are small and have several amphipatic helices in membrane contact, $^{16-18,72}$ both have theoretically a strong curvatureinducting effect, which should be enhanced in Bax by oligomerization. In agreement with this, a peptide derived from Bax helix 5 reduced the energy cost associated with curved membranes. ${ }^{44}$

A second, not mutually exclusive possibility is that Bax stabilizes local membrane curvature by scaffolding. A large part of the structure of membrane-embedded Bax was recently solved. ${ }^{16,17}$ Helices $2-5$ of Bax form a rigid, slightly curved homo-dimer, with many hydrophobic and aromatic amino acids clustered at the interface proposed to be in membrane contact. ${ }^{16-18}$ Assuming this idea is correct, Bax could force its own curved shape onto the membrane as shown for BAR-domain proteins ${ }^{74}$ (Figure 4c).

Finally, we cannot discard that cBid and Bax stabilize local membrane curvature by recruiting lipids with intrinsic curvature that stabilize non-lamellar membrane structures. ${ }^{35,36,68,75}$ Cardiolipin is a cone-shaped lipid that is important for membrane binding of $\mathrm{cBid}$ and Bax. ${ }^{24,76,77}$ The preferential interaction of $\mathrm{cBid}$ and Bax with this lipid could increase its local concentration and reduce the energy required for membrane remodeling.

In addition, our results demonstrate that $\mathrm{Bcl}-\mathrm{xL}$ inhibits not only cBid/Bax induced pore formation but also vesicle budding and fission. Bcl-xL modulates Bax via different mechanisms: (i) $\mathrm{Bcl}-\mathrm{xL}$ inhibits Bax activation by sequestering tBid; ${ }^{24,26}$ (ii) $\mathrm{BCl}-\mathrm{xL}$ reduces the oligomerization state of $\mathrm{Bax}$ within membranes; ${ }^{78}$ and (iii) $\mathrm{Bcl}-\mathrm{xL}$ decreases the membranebound population of Bax. ${ }^{11-13}$ In addition, our data support a novel mechanism by which $\mathrm{Bcl}-\mathrm{xL}$ inhibits the activity of $\mathrm{cBid}$ and Bax by restraining their effects on membrane curvature. cBid alone is able to affect membrane structure and this activity is lost when $\mathrm{Bcl}-\mathrm{xL}$ is present. As tBid adopts different conformations in the membrane ${ }^{22,79}$ and forms tight complexes with $\mathrm{Bcl}-\mathrm{xL},{ }^{26}$ it is tempting to speculate that, in complex with $\mathrm{Bcl}-\mathrm{xL}, \mathrm{cBid}$ and maybe even Bax capture conformations unable to induce membrane curvature. In any case, our findings reveal that $\mathrm{Bcl}-\mathrm{xL}$ inhibits apoptosis by more complex mechanisms than thought so far.

The membrane remodeling activity described here for $\mathrm{cBid}$ and Bax may have physiological implications in the context of the cell. It was shown that tBid translocates to mitochondrial contact sites and reorganizes the mitochondrial structure at the cristae junctions and the contacts between inner and outer mitochondrial membranes. ${ }^{80}$ The ability of cBid to reorganize membrane shape reported here correlates very well with this.

Several studies report that Bax co-localizes at the MOM with Drp1 and Mitofusins that are involved in mitochondrial dynamics. Moreover, Bax is proposed to be involved in the regulation of mitochondrial dynamics. ${ }^{28-30,40}$ The generation of local high curvature and the stabilization of non-lamellar membrane intermediates by Bax at the MOM fission and fusion sites could reduce the energy required for Drp1 and/or mitofusin-mediated membrane fission and fusion, thus promoting these processes. Given that the intrinsic pathway of apoptosis is accompanied by massive mitochondrial fragmentation mediated by Drp1, it is tempting to speculate that Bax could act as a cofactor of Drp1 to promote mitochondrial fission.

In summary, our work reveals that cBid and Bax are not only involved in membrane permeabilization but can also induce membrane remodeling. We propose that this is achieved mechanically by inducing or stabilizing membrane curvature, which supports a common molecular basis for their role in MOM permeabilization and in mitochondrial dynamics. In line with its function as cBid/Bax antagonist, Bcl-xL can inhibit membrane remodeling. Future research is needed to understand the complex molecular interplay between $\mathrm{Bcl}-2$ proteins and the machinery regulating mitochondrial dynamics.

\section{Materials and Methods}

Protein production and labeling. Full-length mouse Bid, full-length human Bax and full-length human Bcl-xL were expressed in E. coli and purified as described in Bleicken et al. ${ }^{15,24}$ and Suzuki et al. ${ }^{81}$ From Bid, cBid was cleaved and purified as described in Bleicken et al. ${ }^{14}$ Bovine Cyt $c$ and Allophycocyanin (APC) were purchased at Sigma-Aldrich (Munich, Germany). Protein labeling was performed as described in Bleicken et al. ${ }^{24,43}$ Human Drp1 was produced as a fusion protein with the calmodulin-binding protein as described in Ugarte-Uribe et al. ${ }^{51}$ GTPase activity and membrane binding of Drp1 were shown in Ugarte-Uribe et al. ${ }^{51}$

Composition of the lipid mixtures. The lipid mixture mimicking the MOM composition was prepared as in ${ }^{14,16,21}$ with $49 \%$ egg L- $\alpha$-phosphatidyl-choline, $27 \%$ egg $L-\alpha$ phosphatidyl-ethanolamine, $10 \%$ bovine liver $L-\alpha$-phosphatidyl-inositol, 10\% 18: 1 phosphatidyl-serine and $4 \%$ cardiolipin (all percentages $\mathrm{mol} / \mathrm{mol}$ ). In addition, a lipid mixture composed of $30 \%$ cardiolipin and $70 \%$ phosphatidyl-choline $(\mathrm{mol} / \mathrm{mol})$ was used. ${ }^{24,43}$ All lipids were purchased from Avanti polar lipids (Alabaster, AL, USA). The lipidic dyes DiD (1,1'-dioctadecyl-3,3,3',3'-tetramethylindodicarbocyanine, 4-chlorobenzenesulfonate), Dil (1,1'-dioctadecyl-3,3,3',3'-tetramethylindocarbocyanine perchlorate) and DiO (3,3'-dilinoleyloxacarbocyanine perchlorate) (all Thermo Fisher Scientific, Waltham, MA, USA) were used to visualize membranes in the confocal microscope.

LUV preparation. The dried lipid mixtures was dissolved in buffer $(150 \mathrm{mM}$ $\mathrm{NaCl}, 20 \mathrm{mM}$ tris $\mathrm{pH} 7.5$ ) and prepared as described in Bleicken et al. ${ }^{14,15}$ Briefly, mixing of the lipid film and buffer was accompanied by five cycles of freezing and thawing. Afterwards the lipid solution was passed 31 times through a membrane (200 or $400 \mathrm{~nm}$ pore size) using an extruder from Avestin (Mannheim, Germany).

GUV permeabilization and GUV size determination experiments. GUVs were produced by electroformation and the experiments were done as described in Bleicken et al..$^{24,82}$ Briefly, $10 \mu \mathrm{g}$ lipid mixture dissolved in chloroform were spread on platinum electrodes in the electroformation chamber and allowed to dry, before immersion in $300 \mathrm{mM}$ sucrose. Electroformation proceeded for $2 \mathrm{~h}$ at $10 \mathrm{~Hz}$, followed by $30 \mathrm{~min}$ at $2 \mathrm{~Hz}$. Cyt $\mathrm{C}_{488}, \mathrm{APC}$ and the proteins of interest were mixed in LabTec chambers (Thermo Fisher Scientific) with buffer ( $150 \mathrm{mM} \mathrm{NaCl}$, $20 \mathrm{mM}$ tris, $\mathrm{pH}$ 7.5) at the desired concentrations. Afterwards $75 \mu \mathrm{l}$ of the GUVs suspension was added to get a final volume of $300 \mu \mathrm{l}$. Imaging was performed after 45-90 min incubation at room temperature, as indicated in the figure. The samples were imaged using a LSM710 confocal microscope with a C-Apochromat $\times 40 \mathrm{NA}$. 1.2 water immersion objective (Zeiss, Jena, Germany) with laserlines to excitate at 488,561 or $633 \mathrm{~nm}$. A spectral beam guide was used to separate emitted fluorescence. Images were processed with ImageJ (http://rsbweb.nih.gov/ij) or a 
homemade analysis software ${ }^{48}$ detecting the filling and the size of each GUVs. Per sample well 200-1500 GUVs were analyzed.

The degree of GUV filling was calculated as:

$\%$ GUV filling $=\left[\left(F_{t}^{\text {in }}-F_{0}\right) /\left(F_{t}^{\text {out }}-F_{0}\right)\right] \times 100$

where $F_{t}^{\text {in }}$ and $F_{t}^{\text {ut }}$ are the average fluorescence intensities inside and outside a GUV at time $t$, and $F_{0}$ is the background fluorescence. We arbitrarily set the threshold for classifying GUVs as non-permeabilized for $<20 \%$. The experiments were set up as a way that the lipid to Bax ratio was $\sim 500: 1$ or bigger. To make sure that the samples were statistically different, $P$-values were calculated by performing $t$-tests using the Graph Pad prism software.

\section{Fluorescence correlation spectroscopy experiments on LUVs and fluorescence burst analysis on GUVs}

Experiments on LUVs: LUVs mimicking the MOM composition (and $<0.05 \%$ DiD) were incubated with Bcl-2 proteins for $120 \mathrm{~min}$ at RT before solution FCS experiments were performed using a LSM710 confocal microscope equipped with a Confocor3 for FCS measurements, a C-Apochromat $\times 40$ NA. 1.2 water immersion objective and laser to excite at $633 \mathrm{~nm}$ (Zeiss). The protein concentrations were chosen so that the lipid to Bax ratio was at least $500: 1$. Each sample was measured at least $300 \mathrm{~s}$ to assure sufficient data points to generate auto-correlation curves. To calculate the $t_{\mathrm{D}}$, the data were fitted using equations 2 and 3 assuming three-dimensional diffusion.

Auto-correlation function $G(\tau)=\frac{\langle\delta F(t) \delta F(t+\tau)\rangle}{\langle F(t)\rangle^{2}}$

3D diffusion $\mathrm{G}_{3 \mathrm{D}}(\tau)=\frac{1}{N}\left(1+\frac{\tau}{\tau_{\mathrm{D}}}\right)^{-1} \frac{1}{\sqrt{1+\frac{\tau}{S^{2} \tau_{\mathrm{D}}}}}$

Experiments on GUVs. For the burst analysis, the LSM710 with Confocor3 was used as mentioned earlier. GUVs mimicking the composition of the MOM were incubated at RT with Bcl-2 proteins in the absence of size marker proteins (to avoid noise due to channel cross talk). After incubation, images were taken to detect the mean GUV size. Moreover, fluorescence intensity traces were collected at three different position of the sample (each for $200 \mathrm{~s}$ and $60-80 \mu \mathrm{m}$ above the glass; laser power: $0.5 \%$; pinhole: 2 Airy units; [Dil]: $0.02 \%$ ). Thereby, 600000 data points per sample and experiment were collected. From the traces, the number of peaks with fluorescence intensity as bigger as 10-fold than the background intensity (mean count rate background: $\sim 3 \mathrm{kHz}$ ) were counted and compared for different samples. More information is given in the Supplementary Information.

Dynamic light scattering experiments. DLS experiments were performed on a zetasizer instrument (Malvern, Malvern, UK). Samples contain vesicles ( $20 \mu \mathrm{M}$ lipids) with or without $20 \mathrm{nM} \mathrm{cBid}$ and $40 \mathrm{nM}$ Bax (attenuation 9 or 10 , 10-16 runs per measurement). Experiments were done at RT.

Cryo EM experiments. For sample preparation vesicles (1 $\mathrm{mM}$ lipids) were incubated at $37^{\circ} \mathrm{C}$ with $1 \mu \mathrm{M} \mathrm{cBid}$ and $2 \mu \mathrm{M}$ Bax for $10 \mathrm{~s}$ to $60 \mathrm{~min}$ and afterwards $5 \mu \mathrm{l}$ of the sample was transferred on before glow discharged (3s) 2/2 Quantifoil grids. Grids with sample droplets were blotted and frozen using FEI Vitrobot (Hillsboro, OR, USA) at $4{ }^{\circ} \mathrm{C}$ and $100 \%$ humidity for 8-10 s. The grids were observed in a Titan Krios microscope (FEI) equipped with a Quantum 963 SE energy filter operated at $200 \mathrm{kV}$ and LN temperature. Zero-loss filtered pictures were taken at $\times 64000$ magnification using a 2 k Ultrascan Quantum CCD camera.

Images were taken using the leginon software in a semi-automated procedure. After obtaining a grid map $(x 135)$ selected squares were imaged $(x 480)$ and a group of holes were selected for imaging at $\times 4300$. At that magnification liposomes were visible and 1-4 positions were chosen for final acquisition. Pictures were taken with a dose of $\sim 10 \mathrm{e}-/ \mathrm{A}^{2}$ at a defocus of $\sim 1 \mu \mathrm{m}$.

\section{Conflict of Interest}

The authors declare no conflict of interest.

Acknowledgements. We thank $C$ Stegmüller for excellent technical assistance. The work of SB, BUU and AJGS was supported by Max Planck Society, the
German Ministry for Education and Research (BMBF, grant no. 0312040), DFG grant FOR2036 and European Research Council (ERC-2012-StG-309966).

1. Strasser A, O'Connor L, Dixit VM. Apoptosis signaling. Annu Rev Biochem 2000; 69: 217-245.

2. Rathmell JC, Thompson CB. Pathways of apoptosis in lymphocyte development, homeostasis, and disease. Cell 2002; 109 Suppl: S97-107.

3. Meier P, Finch A, Evan G. Apoptosis in development. Nature 2000; 407: 796-801.

4. Czabotar PE, Lessene G, Strasser A, Adams JM. Control of apoptosis by the BCL-2 protein family: implications for physiology and therapy. Nat Rev Mol Cell Biol 2014; 15 : 49-63.

5. Martinou J-C, Youle RJ. Mitochondria in apoptosis: Bcl-2 family members and mitochondrial dynamics. Dev Cell 2011; 21: 92-101.

6. Youle RJ, Karbowski M. Mitochondrial fission in apoptosis. Nat Rev Mol Cell Biol 2005; 6 : 657-663.

7. Garcia-Saez AJ. The secrets of the Bcl-2 family. Cell Death Differ 2012; 19: 1733-1740.

8. Jourdain A, Martinou JC. Mitochondrial outer-membrane permeabilization and remodelling in apoptosis. Int J Biochem Cell Biol 2009; 41: 1884-1889.

9. Landes T, Martinou JC. Mitochondrial outer membrane permeabilization during apoptosis: the role of mitochondrial fission. Biochim Biophys Acta 2011; 1813: 540-545.

10. Youle RJ, Strasser A. The BCL-2 protein family: opposing activities that mediate cell death. Nat Rev Mol Cell Biol 2008; 9: 47-59.

11. Todt F, Cakir Z, Reichenbach F, Youle RJ, Edlich F. The C-terminal helix of Bcl-xL mediates Bax retrotranslocation from the mitochondria. Cell Death Differ 2013; 20: 333-342.

12. Edlich F, Banerjee S, Suzuki M, Cleland MM, Arnoult D, Wang C et al. Bcl-x(L) Retrotranslocates Bax from the mitochondria into the cytosol. Cell 2011; 145: 104-116.

13. Schellenberg B, Wang P, Keeble JA, Rodriguez-Enriquez R, Walker S, Owens TW et al. Bax exists in a dynamic equilibrium between the cytosol and mitochondria to control apoptotic priming. Molecular Cell 2013; 49: 959-971.

14. Bleicken S, Garcia-Saez AJ, Conte E, Bordignon E. Dynamic interaction of cBid with detergents, liposomes and mitochondria. PLoS One 2012; 7: e35910.

15. Bleicken S, Classen M, Padmavathi PV, Ishikawa T, Zeth K, Steinhoff HJ et al. Molecular details of Bax activation, oligomerization, and membrane insertion. J Biol Chem 2010; 285: $6636-6647$.

16. Bleicken S, Jeschke G, Stegmueller C, Salvador-Gallego R, García-Sáez AJ, Bordignon E et al. Structural Model of Active Bax at the Membrane. Molecular Cell 2014; 56: 496-505.

17. Czabotar PE, Westphal D, Dewson G, Ma S, Hockings C, Fairlie WD et al. Bax crystal structures reveal how $\mathrm{BH} 3$ domains activate Bax and nucleate its oligomerization to induce apoptosis. Cell 2013; 152: 519-531.

18. Westphal D, Dewson G, Menard M, Frederick P, lyer S, Bartolo R et al. Apoptotic pore formation is associated with in-plane insertion of Bak or Bax central helices into the mitochondrial outer membrane. Proc Natl Acad Sci USA 2014; 111: E4076-E4085.

19. Annis MG, Soucie EL, Dlugosz PJ, Cruz-Aguado JA, Penn LZ, Leber B et al. Bax forms multispanning monomers that oligomerize to permeabilize membranes during apoptosis. EMBO J 2005; 24: 2096-2103.

20. Dlugosz PJ, Billen LP, Annis MG, Zhu W, Zhang Z, Lin J et al. Bcl-2 changes conformation to inhibit Bax oligomerization. EMBO J 2006; 25: 2287-2296.

21. Lovell JF, Billen LP, Bindner S, Shamas-Din A, Fradin C, Leber B et al. Membrane binding by tBid initiates an ordered series of events culminating in membrane permeabilization by Bax. Cell 2008; 135: 1074-1084.

22. Shamas-Din A, Bindner S, Zhu W, Zaltsman Y, Campbell C, Gross A et al. tBid undergoes multiple conformational changes at the membrane required for Bax activation. J Biol Chem 2013; 288: 22111-22127.

23. Wolter KG, Hsu YT, Smith CL, Nechushtan A, Xi XG, Youle R. Movement of Bax from the cytosol to mitochondria during apoptosis. J Cell Biol 1997; 139: 1281-1292.

24. Bleicken S, Wagner C, García-Sáez, Ana J. Mechanistic differences in the membrane activity of Bax and Bcl-xL Correlate with their opposing roles in apoptosis. Biophys $\mathrm{J} 2013 ; 104$ : $421-431$.

25. Antonsson B, Montessuit S, Sanchez B, Martinou JC. Bax is present as a high molecular weight oligomer/complex in the mitochondrial membrane of apoptotic cells. J Biol Chem 2001; 276: 11615-11623.

26. Garcia-Saez AJ, Ries J, Orzaez M, Perez-Paya E, Schwille P. Membrane promotes tBID interaction with $\mathrm{BCL}(\mathrm{XL})$. Nat Struct Mol Biol 2009; 16: 1178-1185.

27. Llambi F, Moldoveanu T, Tait SW, Bouchier-Hayes L, Temirov J, McCormick LL et al. A unified model of mammalian Bcl-2 protein family interactions at the mitochondria. Mol Cell 2011; 44: 1-15.

28. Karbowski M, Lee YJ, Gaume B, Jeong SY, Frank S, Nechushtan A et al. Spatial and temporal association of Bax with mitochondrial fission sites, Drp1, and Mfn2 during apoptosis. J Cell Biol 2002; 159: 931-938.

29. Karbowski M, Norris KL, Cleland MM, Jeong SY, Youle RJ. Role of Bax and Bak in mitochondrial morphogenesis. Nature 2006; 443: 658-662.

30. Karbowski M, Youle RJ. Dynamics of mitochondrial morphology in healthy cells and during apoptosis. Cell Death Differ 2003; 10: 870-880. 
31. Berman SB, Chen YB, Qi B, McCaffery JM, Rucker EB 3rd, Goebbels $S$ et al. Bcl-xL increases mitochondrial fission, fusion, and biomass in neurons. J Cell Biol 2009; 184: 707-719.

32. Karbowski M, Arnoult D, Chen H, Chan DC, Smith CL, Youle RJ. Quantitation of mitochondrial dynamics by photolabeling of individual organelles shows that mitochondrial fusion is blocked during the Bax activation phase of apoptosis. J Cell Biol 2004; 164: 493-499.

33. Li H, Alavian KN, Lazrove E, Mehta N, Jones A, Zhang P et al. A Bcl-XL-Drp1 complex regulates synaptic vesicle membrane dynamics during endocytosis. Nat Cell Biol 2013; 15 773-785.

34. Li H, Chen Y, Jones AF, Sanger RH, Collis LP, Flannery R et al. Bcl-xL induces Drp1dependent synapse formation in cultured hippocampal neurons. Proc Natl Acad Sci USA 2008; 105: 2169-2174

35. Frolov VA, Zimmerberg J. Cooperative elastic stresses, the hydrophobic effect, and lipid tilt in membrane remodeling. FEBS Lett 2010; 584: 1824-1829.

36. Kozlov MM, Campelo F, Liska N, Chernomordik LV, Marrink SJ, McMahon HT. Mechanisms shaping cell membranes. Curr Opin Cell Biol 2014; 29: 53-60.

37. Kozlov MM, McMahon HT, Chernomordik LV. Protein-driven membrane stresses in fusion and fission. Trends Biochem Sci 2010; 35: 699-706.

38. Chernomordik LV, Kozlov MM. Mechanics of membrane fusion. Nat Struct Mol Biol 2008; 15 675-683.

39. Terrones $\mathrm{O}$, Antonsson B, Yamaguchi $\mathrm{H}$, Wang $\mathrm{HG}$, Liu J, Lee RM et al. Lipidic pore formation by the concerted action of proapoptotic BAX and tBID. J Biol Chem 2004; 279 30081-30091.

40. Montessuit S, Somasekharan SP, Terrones O, Lucken-Ardjomande S, Herzig S, Schwarzenbacher $\mathrm{R}$ et al. Membrane remodeling induced by the dynamin-related protein Drp1 stimulates Bax oligomerization. Cell 2010; 142: 889-901.

41. Basanez G, Nechushtan A, Drozhinin O, Chanturiya A, Choe E, Tutt S et al. Bax, but not Bcl$\mathrm{x}(\mathrm{L})$, decreases the lifetime of planar phospholipid bilayer membranes at subnanomolar concentrations. Proc Natl Acad Sci USA 1999; 96: 5492-5497.

42. Qian S, Wang W, Yang L, Huang HW. Structure of transmembrane pore induced by Baxderived peptide: evidence for lipidic pores. Proc Natl Acad Sci USA 2008; 105 17379-17383.

43. Bleicken S, Landeta O, Landajuela A, Basanez G, Garcia-Saez AJ. Proapoptotic Bax and Bak form stable protein-permeable pores of tunable size. J Biol Chem 2013; 288 33241-33252.

44. Garcia-Saez AJ, Chiantia S, Salgado J, Schwille P. Pore formation by a Bax-derived peptide: effect on the line tension of the membrane probed by AFM. Biophys J 2007; 93: 103-112.

45. Fuertes G, Gimenez D, Esteban-Martin S, Sanchez-Munoz OL, Salgado J. A lipocentric view of peptide-induced pores. Eur Biophys J Biophys Lett 2011; 40: 399-415.

46. Ros U, Garcia-Saez AJ. More than a pore: the interplay of pore-forming proteins and lipid membranes. J Membr Biol 2015; 248: 545-561.

47. Billen LP, Kokoski CL, Lovell JF, Leber B, Andrews DW. Bcl-XL inhibits membrane permeabilization by competing with Bax. PLoS Biol 2008; 6: e147.

48. Hermann E, Bleicken S, Subburaj Y, Garcia-Saez AJ. Automated analysis of giant unilamellar vesicles using circular Hough transformation. Bioinformatics 2014; 30: 1747-1754.

49. Ugarte-Uribe B, Garcia-Saez AJ. Membranes in motion: mitochondrial dynamics and their role in apoptosis. Biol Chem 2014; 395: 297-311.

50. Oettinghaus B, Licci M, Scorrano L, Frank S. Less than perfect divorces: dysregulated mitochondrial fission and neurodegeneration. Acta Neuropathol 2012; 123: 189-203.

51. Ugarte-Uribe B, Mueller HM, Otsuki M, Nickel W, Garcia-Saez AJ. Dynamin-related protein 1 (Drp1) promotes structural intermediates of membrane division. J Biol Chem 2014; 289: 30645-30656.

52. Cassidy-Stone A, Chipuk JE, Ingerman E, Song C, Yoo C, Kuwana T et al. Chemical inhibition of the mitochondrial division dynamin reveals its role in Bax/Bak-dependent mitochondrial outer membrane permeabilization. Dev Cell 2008; 14: 193-204.

53. Tanaka A, Youle RJ. A chemical inhibitor of DRP1 uncouples mitochondrial fission and apoptosis. Mol Cell 2008; 29: 409-410.

54. Pagliari LJ, Kuwana T, Bonzon C, Newmeyer DD, Tu S, Beere HM et al. The multidomain proapoptotic molecules Bax and Bak are directly activated by heat. Proc Natl Acad Sci USA 2005; 102: 17975-17980.

55. Lorenz M, Vollmer B, Unsay JD, Klupp BG, García-Sáez AJ, Mettenleiter TC et al. A single herpesvirus protein can mediate vesicle formation in the nuclear envelope. $J$ Biol Chem 2015; 290: 6962-6974.

56. Drucker P, Pejic M, Galla HJ, Gerke V. Lipid segregation and membrane budding induced by the peripheral membrane binding protein annexin A2. J Biol Chem 2013; 288: 24764-24776.

57. Wollert T, Hurley JH. Molecular mechanism of multivesicular body biogenesis by ESCRT complexes. Nature 2010; 464: 864-869

58. Landeta O, Landajuela A, Gil D, Taneva S, Di Primo C, Sot B et al. Reconstitution of proapoptotic BAK function in liposomes reveals a dual role for mitochondrial lipids in the BAK-driven membrane permeabilization process. J Biol Chem 2011; 286: 8213-8230.
59. Schafer B, Quispe J, Choudhary V, Chipuk JE, Ajero TG, Du H et al. Mitochondrial outer membrane proteins assist Bid in Bax-mediated lipidic pore formation. Mol Biol Cell 2009; 20 2276-2285.

60. Gillies LA, Du H, Peters B, Knudson CM, Newmeyer DD, Kuwana T. Visual and functional demonstration of growing Bax-induced pores in mitochondrial outer membranes. Mol Bio Cell 2015; 26: 339-349.

61. Hernandez JM, Stein A, Behrmann E, Riedel D, Cypionka A, Farsi Z et al. Membrane fusion intermediates via directional and full assembly of the SNARE complex. Science 2012; 336 1581-1584

62. Diao J, Grob P, Cipriano DJ, Kyoung M, Zhang Y, Shah S et al. Synaptic proteins promote calcium-triggered fast transition from point contact to full fusion. Elife 2012; 1: e00109.

63. Youle RJ, van der Bliek AM. Mitochondrial fission, fusion, and stress. Science 2012; 337 1062-1065.

64. Kuwana T, Mackey MR, Perkins G, Ellisman MH, Latterich M, Schneiter R et al. Bid, Bax, and lipids cooperate to form supramolecular openings in the outer mitochondrial membrane. Cell 2002; 111: 331-342.

65. Antonsson B, Montessuit S, Lauper S, Eskes R, Martinou JC. Bax oligomerization is required for channel-forming activity in liposomes and to trigger cytochrome $\mathrm{C}$ release from mitochondria. Biochem J 2000; 345: 271-278.

66. Boucrot E, Pick A, Camdere G, Liska N, Evergren E, McMahon HT et al. Membrane fission is promoted by insertion of amphipathic helices and is restricted by crescent BAR domains. Cell 2012; 149: 124-136.

67. Renard HF, Simunovic M, Lemière J, Boucrot E, Garcia-Castillo MD, Arumugam S et al. Endophilin-A2 functions in membrane scission in clathrin-independent endocytosis. Nature 2015; 517: 493-496.

68. McMahon HT, Boucrot E. Membrane curvature at a glance. J Cell Sci2015; 128: 1065-1070.

69. Jahn R, Fasshauer D. Molecular machines governing exocytosis of synaptic vesicles. Nature 2012; 490: 201-207.

70. Vrljic M, Strop P, Ernst JA, Sutton RB, Chu S, Brunger AT et al. Molecular mechanism of the synaptotagmin-SNARE interaction in $\mathrm{Ca2}+$-triggered vesicle fusion. Nat Struct $\mathrm{Mol}$ Biol 2010; 17: 325-331.

71. Zhou Q, Lai Y, Bacaj T, Zhao M, Lyubimov AY, Uervirojnangkoorn $M$ et al. Architecture of the synaptotagmin-SNARE machinery for neuronal exocytosis. Nature 2015; 525: 62-67.

72. Wang $Y$, Tjandra N. Structural insights of tBid, the caspase-8-activated Bid, and its BH3 domain. J Biol Chem 2013; 288: 35840-35851.

73. Stachowiak JC, Brodsky FM, Miller EA. A cost-benefit analysis of the physical mechanisms of membrane curvature. Nat Cell Biol 2013; 15: 1019-1027.

74. Zimmerberg J, Kozlov MM. How proteins produce cellular membrane curvature. Nat Rev Mol Cell Biol 2006; 7: 9-19.

75. Frolov VA, Shnyrova AV, Zimmerberg J. Lipid polymorphisms and membrane shape. Cold Spring Harb Perspect Biol 2011; 3: a004747.

76. Shamas-Din A, Bindner S, Chi X, Leber B, Andrews DW, Fradin C. Distinct lipid effects on tBid and Bim activation of membrane permeabilization by pro-apoptotic Bax. Biochem $J$ 2015; 467: 495-505.

77. Lutter M, Fang M, Luo X, Nishijima M, Xie X, Wang X. Cardiolipin provides specificity for targeting of tBid to mitochondria. Nat Cell Biol 2000; 2: 754-761.

78. Subburaj Y, Cosentino K, Axmann M, Pedrueza-Villalmanzo E, Hermann E, Bleicken S et al. Bax monomers form dimer units in the membrane that further self-assemble into multiple oligomeric species. Nat Commun 2015; 6: 8042.

79. Shamas-Din A, Satsoura D, Khan O, Zhu W, Leber B, Fradin C et al. Multiple partners can kiss-and-run: Bax transfers between multiple membranes and permeabilizes those primed by tBid. Cell Death Dis 2014; 5 : e1277.

80. Scorrano L, Ashiya M, Buttle K, Weiler S, Oakes SA, Mannella CA et al. A distinct pathway remodels mitochondrial cristae and mobilizes cytochrome c during apoptosis. Dev Cell 2002; 2: $55-67$.

81. Suzuki M, Youle RJ, Tjandra N. Structure of Bax: coregulation of dimer formation and intracellular localization. Cell 2000; 103: 645-654.

82. Bleicken S, Garcia-Saez AJ. New biophysical methods to study the membrane activity of bcl2 proteins. Methods Mol Biol 2014; 1176: 191-207.

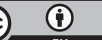

Cell Death and Disease is an open-access journal published by Nature Publishing Group. This work is licensed under a Creative Commons Attribution 4.0 International License. The images or other third party material in this article are included in the article's Creative Commons license, unless indicated otherwise in the credit line; if the material is not included under the Creative Commons license, users will need to obtain permission from the license holder to reproduce the material. To view a copy of this license, visit http://creativecommons.org/licenses/by/4.0/ 